\title{
Two-Dimensional Interleaving Schemes with Repetitions
}

\author{
Mario Blaum \\ IBM Almaden Research Center \\ 650 Harry Rd. \\ San Jose, CA 95120 \\ blaumalmaden.ibm.com
}

\author{
Jehoshua Bruck* \\ California Institute of Technology \\ MS 136-93 \\ Pasadena, CA 91125 \\ bruckeparadise.caltech.edu
}

\author{
Patrick G. Farrell \\ Electrical Engineering Laboratories \\ The University, Manchester \\ M13 9PL, England \\ farrelloman.ac.uk
}

\begin{abstract}
We present 2-dimensional interleaving schemes, with repetition, for correcting 2-dimensional bursts (or clusters) of errors, where a cluster of errors is characterized by its area. Known interleaving schemes are based on arrays of integers with the property that every connected component of area $t$ consists of distinct integers. Namely, they are based on the use of 1-error-correcting codes. We extend this concept by allowing repetitions within the arrays, hence, providing a trade-off between the error-correcting capability of the codes and the degree of the interleaving schemes.
\end{abstract}

Most 2-dimensional burst error-correcting codes that have been studied in the literature so far consider burst errors of a given shape, including rectangular and circular shapes $[1,4,5,6]$. Here we present 2-dimensional interleaving schemes for correcting 2-dimensional bursts (or clusters) of errors, where a cluster of errors is characterized by its area. A recent application of correction of 2-dimensional clusters appeared in the context of holographic storage [7]. In [2] we presented interleaving schemes that are based on arrays of integers with the property that every connected component of area $t$ consists of distinct integers. These arrays are called $t$-interleaved arrays. Here, we extend the concept of $t$-interleaved arrays by allowing repetitions within the arrays. Namely, a $t$-interleaved array with repetition $r$ is an array of integers with the property that in every connected component of area $t$, every integer is repeated at most $r$ times.

Definition 1 We say that a set of $t$ elements in a 2 dimensional array is a cluster of size $t$, if any two elements in the cluster belong in a path contained in the set.

Definition 2 Let $t \geq 1$ and $r \geq 1$ be integers. Let $A(t, r)$ be a 2-dimensional array of integers, namely, the elements of the array are labeled by integers. We say that $A(t, r)$ is $t$ interleaved with repetition $r$ if every cluster of size $t$ in $A(t, r)$ consists of integers that repeat at most $r$ times. The degree of interleaving of the array is the number of distinct integers it contains.

For example, the following is an $A(5,2)$ array with degree of interleaving 5 . In fact, this is the optimal degree:

$A(5,2)=$\begin{tabular}{|l|l|l|l|l|l|l|l|l|l|}
\hline 0 & 1 & 2 & 3 & 4 & 0 & 1 & 2 & 3 & 4 \\
\hline 2 & 3 & 4 & 0 & 1 & 2 & 3 & 4 & 0 & 1 \\
\hline 4 & 0 & 1 & 2 & 3 & 4 & 0 & 1 & 2 & 3 \\
\hline 1 & 2 & 3 & 4 & 0 & 1 & 2 & 3 & 4 & 0 \\
\hline 3 & 4 & 0 & 1 & 2 & 3 & 4 & 0 & 1 & 2 \\
\hline
\end{tabular}

- Supported in part by the NSF Young Investigator Award CCR-9457811, by the Sloan Research Fellowship and by DARPA and BMDO through an agreement with NASA/OSAT.

\begin{tabular}{|c|c|c|}
\hline$t$ & Lower bound & Upper bound \\
\hline 3 & 2 & 2 \\
4 & 3 & 3 \\
5 & 5 & 5 \\
6 & 7 & 7 \\
7 & 8 & 10 \\
8 & 10 & 12 \\
9 & 13 & 16 \\
10 & 15 & 19 \\
11 & 18 & 22 \\
12 & 21 & 27 \\
\hline
\end{tabular}

Table 1: Lower and upper bounds on the degree of interleaving of $A(t, 2)$.

Notice that, if the integers represent different codes (like in the one-dimensional case), then $r$-error-correcting codes distributed in a $t$-interleaved array with repetition $r$ can correct any cluster of size up to $t$. In the above example, we need only five 2-error correcting codes to correct any cluster of size 5 .

Our goal is to construct $A(t, r)$ arrays with minimal degree. In [2] we presented optimal degree constructions of $A(t, 1)$ arrays for arbitrary $t$. Here we focus on the case $r=2$ and present lower bounds and constructions. Table 1 summarizes, for specific numbers, the lower bounds and the upper bounds on the degree of interleaving of $A(t, 2)$. For more details we refer the reader to [3]

\section{REFERENCES}

[1] K. A. S. Abdel-Ghaffar, R. J. McEliece and H. C. A. van Tilborg, Two-Dimensional Burst Identification Codes and their Use in Burst Correction, IEEE Trans. on Information Theory, IT-34, pp. 494-504, 1988.

[2] M. Blaum and J. Bruck, Interleaving Schemes for Multidimensional Cluster Errors, IBM Research Report, RJ 9984, Oct. 1995

[3] M. Blaum, J. Bruck and P. G. Farrell, Two-Dimensional Interleaving Schemes with Repetitions, IBM Research Report, RJ 10047 , Oct. 1996.

See also http://paradise.caltech.edu/ETR.html.

[4] M. Blaum and P. G. Farrell, Array Codes for Cluster-Error Correction, Electronic Letters, Vol. 30, No. 21, pp. 1752-3, Oct. 1994.

[5] E. M. Gabidulin and V. V. Zanin, Codes Correcting Array Bursts, The Workshop on Information Protection, Proc., pp. 2425, Moscow, 1993.

[6] H. Imai, A Theory of Two Dimensional Cyclic Codes, Information and Control, Vol 34, pp. 1-21, 1977.

[7] S. A. Lis and P. D. Henshaw, Ultra-Dense Optical Mass Storage, Report prepared for USAF, AFSC, AD-A232 767, 1991. 